\title{
Aerodynamic shape optimization of the STARC-ABL concept for minimal inlet distortion
}

\author{
Gaetan K.W. Kenway,* \\ Cetin Kiris ${ }^{\dagger}$ \\ NASA Ames Research Center, Moffett Field, CA, USA
}

\begin{abstract}
The NASA single-aisle turboelectric aircraft with an aft boundary layer propulsor (STARC-ABL) concept utilizes a novel electrically driven aft fan that ingests the fuselage boundary-layer for increased propulsive efficiency. In this paper we examine how aerodynamic shaping of the fuselage diffuser and nacelle inlet can reduce the flow distortion at the aft fan. Adjoint-based aerodynamic shape optimization with the ARP1420 distortion metric objective is used to automatically determine the optimal shapes for minimal fan-face distortion. Single and multipoint optimizations are carried out for simplified body-duct and wing-body-duct configurations. These two configurations highlight the importance of including the wing downwash effects when designing the propulsor. The optimizations showed the body-duct configuration can obtain cruise distortion values of approximately $1 \%$ while the wing-body-duct configuration can obtain distortion values of just over $2 \%$.
\end{abstract}

\section{Introduction}

The overall configuration of subsonic commercial transport aircraft have remained effectively frozen since the introduction of the swept-wing Boeing 707 in the late 1950's. While the configuration of modern aircraft may be the same, decades of sustained innovation in engine and airframe design has continually driven fuel burn per seat-mile to lower and lower values. NASA's Advanced Air Transport Technology Project (AATT) has the long term goal of dramatically reducing fuel burn, emissions and noise even further. To meet these aggressive targets, it may be necessary to consider novel aircraft configurations.

One potential technology that may enable the next-generation of ultra-efficient commercial aircraft is Boundary Layer Ingestion (BLI). The fundamental concept of a propulsion system incorporating BLI is to re-accelerate the slower moving air from the airframe's boundary layer. The amount of thrust produced by a propulsion system, for a given mass-flow rate, is proportional to the change in momentum of the inlet flow. If the density does not vary appreciably, the thrust produced is therefore proportional to the change in velocity. Since the boundary layer air has much lower velocity than the free-stream air, the propulsion system does not need to add as much kinetic energy to the flow to increase the velocity by fixed amount, thereby achieving the same thrust. The lower propulsor kinetic energy requirement translates to lower fuel burn and a more efficient aircraft.

There have been several novel aircraft concepts proposed that tightly integrate BLI into the vehicle design. The MIT D8 double bubble ${ }^{5,24}$ concept places two aft mounted turbofans between a $\pi$ tail, ingesting a substantial portion of the fuselage boundary layer. Many blended wing body (BWB) concept aircraft propose incorporating BLI into the design. ${ }^{16}$ A new concept aircraft known as NOVA ${ }^{27}$ proposes two aftmounted turbofan ingesting the boundary layer from the side of the fuselage. A study by Mikic et al. ${ }^{9}$ considered the benefits of BLI for a small electrically powered commuter aircraft. Other studies such as those by Emiligui et al. ${ }^{6}$ Plas et al. ${ }^{22}$ and Carrier et al. ${ }^{1}$ investigate potential BLI benefits for simplified configurations.

This focuses on another novel aircraft configuration: the single-aisle turboelectric aircraft with an aft boundary layer propulsor (STARC-ABL) concept. This configuration was first proposed by Welstead et al. ${ }^{26}$

\footnotetext{
${ }^{*}$ Computational Aerosciences Branch, gaetan.k.kenway@nasa.gov

${ }^{\dagger}$ Computational Aerosciences Branch, cetin.c.kiris@nasa.gov
} 
The concept uses a conventional aircraft configuration but adds an electrically driven aft fan running on energy extracted from two conventional under-wing mounted turbofan engines. This separation of the power producing components (engines) and thrust producing components (propulsor) is a key factor in obtaining efficiency gains from effective propulsion-airframe integration (PAI). The largest unknown of the proposed turboelectric propulsion system is whether the performance increases that can be obtained from the BLI are sufficient to offset the additional electrical efficiency losses and system weight. The systems-level analysis performed by Welstead et al. ${ }^{26}$ indicated that there is a potential for $7 \%$ to $12 \%$ block fuel burn savings compared to an equivalent technology conventional configuration. In that work, there was no attempt made to model the aft propulsor with higher fidelity methods such as computational fluid dynamics (CFD). There are two benefits of the STARC-ABL configuration from a propulsion-airframe integration perspective: the first is that the turbofan engines only see free-stream air and thus the BLI does not affect the thermodynamic efficiency of the propulsion system. The second is the possibility of much lower inlet distortion compared to other configurations such as the D8. Unlike the other proposed configurations, the propulsor sees essentially radially symmetric inflow conditions. However, since it is necessary to have upsweep on the fuselage, the propulsor cannot be symmetrically placed on the center line of the fuselage. The main goal of this paper is to investigate the potential for minimizing the inlet distortion of the STARC-ABL configuration by asymmetric aerodynamic shaping of the aft fuselage diffuser and nacelle inlet.

\section{Computational Methods}

For this work, we perform aerodynamic shape optimization using the MDO of Aircraft Configuration with High Fidelity $(\mathrm{MACH})$ framework, developed at the University of Michigan. While the framework was designed for aerostructural design optimization studies, ${ }^{13,14}$ we will only be using the aerodynamic optimization capability for this work.

\section{Computational Fluid Dynamics Solver}

The flow solver in MACH is ADflow. ${ }^{19,25}$ For this work, we solve the steady RANS equations on structured, overset meshes. The discretization scheme uses central fluxes with scalar artificial dissipation and the Spalart-Allmaras turbulence model. ${ }^{23}$ Three solution algorithms, diagonalized alternating direction implicit, (DDADI), approximate Newton Krylov (ANK) and Newton-Krylov (NK) are successively applied as the solution convergences to steady state. A discrete adjoint method is implemented by using a combination of reverse-mode automatic differentiation and analytic methods for the efficient computation of the gradients of functions of interest. Lyu et al. ${ }^{18}$ describe the CFD adjoint implementation in more detail. Further details of the overset mesh implementation can be found in Kenway et al. ${ }^{15}$ The aft fan is modeled directly in the CFD solver using the actuator zone technique described by Hall et al. ${ }^{4}$ However, since it is very early in the design study, we do not have detailed information of the fan or stator geometries. Therefore, we simplify the model to apply a uniform thrust over the specified actuator zone with no additional swirl components.

\section{Geometric Parametrization}

For this work we use OpenVSP to parameterize the geometry. ${ }^{10,20}$ OpenVSP provides a convenient way of describing the geometric level of detail present at the conceptual and preliminary design stages of aircraft design. A new code called DVGeometryVSP applies a python-based wrapper around VSP which allows for seamless interoperability with the MACH framework. Specifically, DVGeometryVSP obtains un-intersected discrete quadrilateral surfaces from OpenVSP and projects the CFD surface coordinates onto this discrete surface representation. When the design variables are updated, a new discrete quad surface is obtained and the CFD surface coordinates are re-evaluated from their stored parametric $u, v$ coordinates. Since OpenVSP

does not directly provide surface sensitivities, we resort to finite differencing over the design variables to obtain the sensitivity of the CFD surface coordinates with respect to the VSP design variables.

\section{Mesh Movement}

OpenVSP only provides geometric deformation information for the surface mesh; that is, the part of the volume mesh that lies on the physical wall surface. A separate procedure is then required to propagate the surface perturbations throughout the remainder of the volume mesh. The mesh movement algorithm 
used in this work is an efficient analytic inverse distance method similar to that described by Luke $e t$ al. ${ }^{17}$ Sensitivities required for the adjoint method are provided by a combination of reverse-mode automatic differentiation and analytic methods.

\section{Optimization Algorithm}

The high computational cost of RANS-based optimization demands an optimization algorithm that minimizes the number of function evaluation calls. We use SNOPT (sparse nonlinear optimizer) ${ }^{7}$ with the Python interface pyOpt. ${ }^{21}$ SNOPT is a gradient-based optimizer that implements a sequential quadratic programming method; it is capable of solving large-scale nonlinear optimization problems with thousands of constraints and design variables. SNOPT uses an augmented Lagrangian merit function, and the Hessian of the Lagrangian is approximated using a quasi-Newton method.

\section{Distortion Computation}

The distortion computations are based on the SAE Recommended Practice ARP1420 document. ${ }^{12}$ It provides measures of describing the distortion patterns typically seen in gas turbine inlets. While the descriptions were originally developed to characterize experimental results, with a few slight modifications, it also can serve as an appropriate function for use in gradient based optimization.

ARP $1420^{12}$ describes three parameters used to characterize the distortion for a given radial section of the inlet: intensity, extent and multiple-per-rev. An example of a simple single-per-rev distortion pattern is shown in Figure 1a.

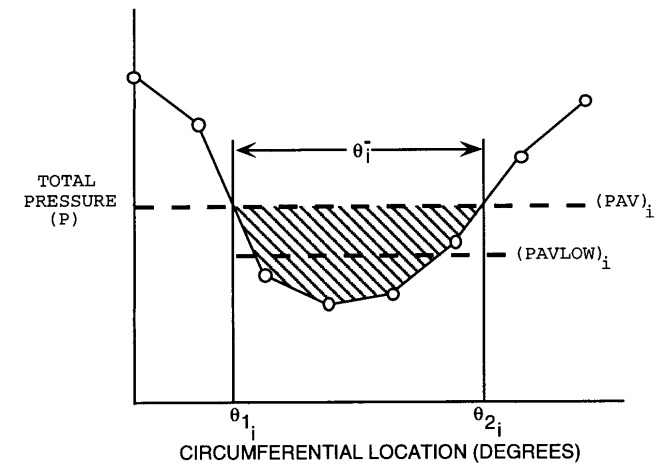

(a) Ring circumferential distortion for once-per-rev pattern.

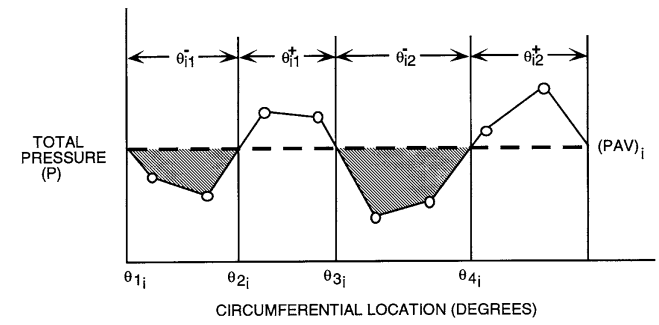

(b) Ring circumferential distortion for multiple-perrev pattern.

Figure 1: Distortion patterns, reproduced from reference. ${ }^{12}$

For this pattern, there is a single low pressure region below the average. The circumferential intensity then defined as

$$
I=\left(\frac{P_{a v g}-P_{a v g_{l o w}}}{P_{a v g}}\right)
$$

where $P_{\text {avg }}$ is the average total pressure on the ring and $P_{a v g_{l o w}}$ is the average total pressure of the region below $P_{\text {avg }}$. The extent of the low pressure region is simply $\theta_{2}-\theta_{1}$ and the multiple-per-rev (MPR) metric is 1 .

For more complex patterns containing multiple low-pressure regions as shown in Figure 1b, the calculation is slightly different.

The calculation is split into two separate categories depending on the circumferential width of the high pressure region separating low pressure regions, $\theta_{i}^{+}$.

$\boldsymbol{\theta}_{\boldsymbol{i}}^{+}<\boldsymbol{\theta}^{+*}$ : If the low pressure regions are separated by only a narrow high pressure region less than $\theta^{+*}$ (taken to be $25^{\circ}$ ) the region is treated as a single-per-rev pattern $(\mathrm{MPR}=1)$ and the intensity is computed using the same as in Eqn 1 but $P_{a v g_{l o w}}$ is computed according to

$$
P_{\text {avg }}=\frac{1}{\theta_{i}^{-}} \sum_{k=1}^{Q} \int_{\theta_{i k}^{-}} P(\theta)_{i} d \theta
$$


where $Q$ is the number of low pressure regions.

$\boldsymbol{\theta}_{\boldsymbol{i}}^{+} \geq \boldsymbol{\theta}^{+*}$ : For distortion patterns separated by a significant extent of higher pressure, the intensity of each low pressure region is computed according to Eqn 1 individually and the overall intensity for this ring is the maximum of the individual regions. The overall extent, is the extent that corresponds to the region of greatest intensity.

Finally, to compute a single optimization function, we perform a smooth Kreisselmeier-Steinhauser $(\mathrm{KS})^{28}$ function aggregation over each of the rings according to:

$$
f=\frac{1}{\rho} \log \left(\sum_{i}^{n} e^{\rho I_{i}}\right)
$$

For this work, $\rho$ was chosen to be 100.0 and $I_{i}$ is intensity of the $i^{\text {th }}$ ring.

Note that a KS function is not employed when taking the maximum intensity within a ring for the multiple-per-rev case. While it initially seems counter-intuitive, a maximum function provides a smoother function than the KS alternative. The issue is that the number of values over which to take the maximum may vary from one for single region pattern up to 4 or more for more complex patterns. For example, at the transition from a single per-rev to two per rev, for a symmetric region, both single and multiple per-rev calculations yield the value for the intensity. However, due to the conservative nature of the KS function once two identical values are considered, a discontinuity is encountered for any finite value of $\rho$. Even through the maximum function may result in "region hopping" ie, the worst region flips between two nearly equally sized regions, this is preferable to the explicit introduction of the KS discontinuity when the number of regions change.

When the ARP1420 distortion metrics are applied in a CFD setting, it is no longer necessary to limit the radial resolution to a low number corresponding to the number of physical probes: we are free to choose any radial resolution up to the mesh resolution. For this work we use a set of 5 equal-area rings with a radial spacing equal to $6^{\circ}$. For each sensor surface, a massflow-averaged total pressure value is computed as opposed to a single centrally located point-based probe. This results in a better representation of the underlying distribution and limits the ability of the optimization algorithm to exploit small, localized effects around a point-based sensor. Figure 2 shows the distribution of the sensor surfaces at the fan-face. The effect of the higher radial resolution in the CFD-based sensor surfaces can be seen in the total pressure contour traces in Figure $7 \mathrm{a}$.

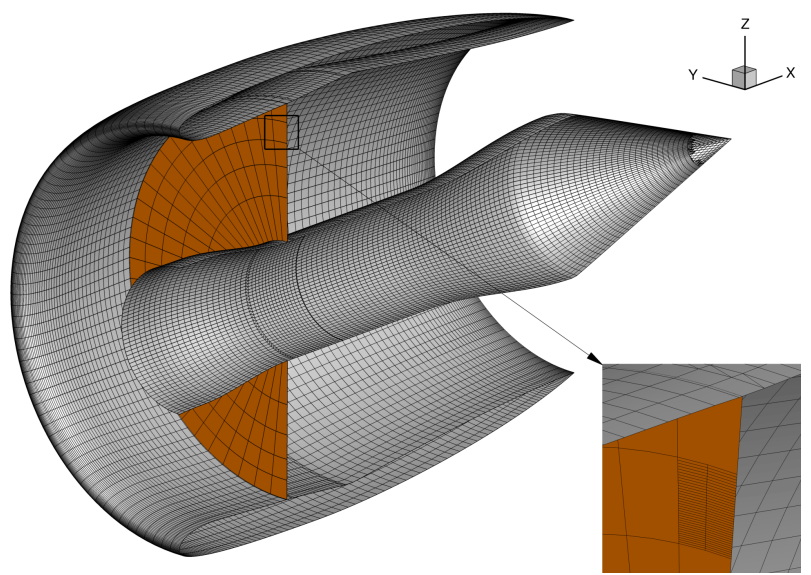

Figure 2: Distribution of sensor surfaces to compute the fan-face distortion metrics. The full resolution of a single sensor surface is shown for reference.

\section{STARC-ABL Geometry}

The baseline model geometry for the STARC-ABL configuration was provided by Jason Welstead from NASA Langley in OpenVSP format. An overview of the full configuration geometry is given in Figure 3. 


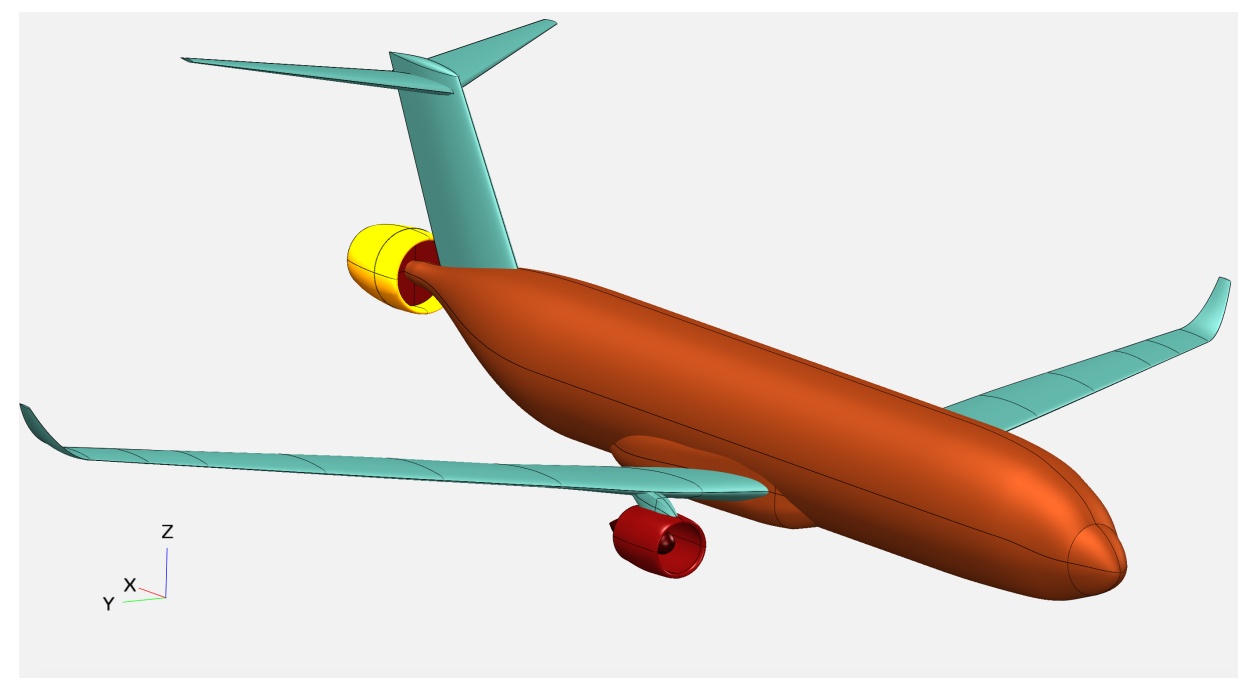

Figure 3: Overview of full STARC-ABL configuration

For this study we are only focusing on the fuselage boundary layer ingestion aspect of the design. However, as will be discussed in the results section, it is critical to include the effect of wing downwash to accurately capture the inlet distortion. In an effort to simplify the analysis we remove the under-wing engines as well as the vertical and horizontal stabilizers. The remaining simplified geometry was meshed using the Chimera Grid Tools ${ }^{2,3}$ package. A series of three meshes with uniformly increasing mesh resolution was generated for the wing-body-duct (wbd) configuration. Solutions were generated for $\alpha=2.0^{\circ}$. The solutions are given in Figure 4. The key mesh characteristics for each of the three meshes is given in Table 1. The mesh sensitivity is low for the two metrics we will be using during the optimization, namely distortion and drag coefficient. The optimizations presented in the results section all use the L1 mesh which is a trade-off between solution accuracy and computational efficiency. The meshes for the body-duct (bd) configuration listed in Table 1 are generated by removing the meshes associated with the wing and belly fairing.

Table 1: Grid characteristics

\begin{tabular}{rlllllll}
\hline Mesh & $\begin{array}{l}\text { Chordwise } \\
\text { cells (wing) }\end{array}$ & $\begin{array}{l}\text { Spanwise } \\
\text { cells (wing) }\end{array}$ & $\begin{array}{l}\text { Circumferential } \\
\text { cells (duct) }\end{array}$ & $\begin{array}{l}y_{\max }^{+} \\
\text {Total } \\
\text { cells }\end{array}$ & $\begin{array}{l}\text { ARP 1420 } \\
\text { Distortion }\end{array}$ & $C_{D}$ (counts) \\
\hline wbd L1 & 92 & 135 & 60 & $\sim 1.47$ & 6075628 & 0.0506 & 246.86 \\
wbd L1.4 & 134 & 188 & 84 & $\sim 0.69$ & 15895100 & 0.0505 & 245.67 \\
wbd L2 & 192 & 266 & 120 & $\sim 0.40$ & 45536903 & 0.0510 & 244.38 \\
\hline bd L1 & - & - & 60 & $\sim 0.37$ & 2925912 & 0.0189 & 96.56 \\
bd L1.4 & - & - & 84 & $\sim 0.26$ & 19248040 & 0.0194 & 96.39 \\
bd L2 & - & - & 120 & $\sim 0.18$ & 22127620 & 0.0201 & 96.36 \\
\hline
\end{tabular}

\section{Optimization Formulation}

The simplified STARC-ABL configuration is parameterized directly using design parameters from the OpenVSP model. A limited set of design variables controlling only the aft fuselage and nacelle inlet are intended to minimize any adverse effects on the remainder of the airframe and propulsion design. Specifically, we do not modify the nacelle geometry aft of the fan. The design variables used in the optimization are graphically shown in Figure 5a. Figure 5b shows thickness constraints that ensure the nacelle does not become excessively thin. Since the nacelle inlet is defined to be an ellipse, only two sets of thickness constraints are necessary. 


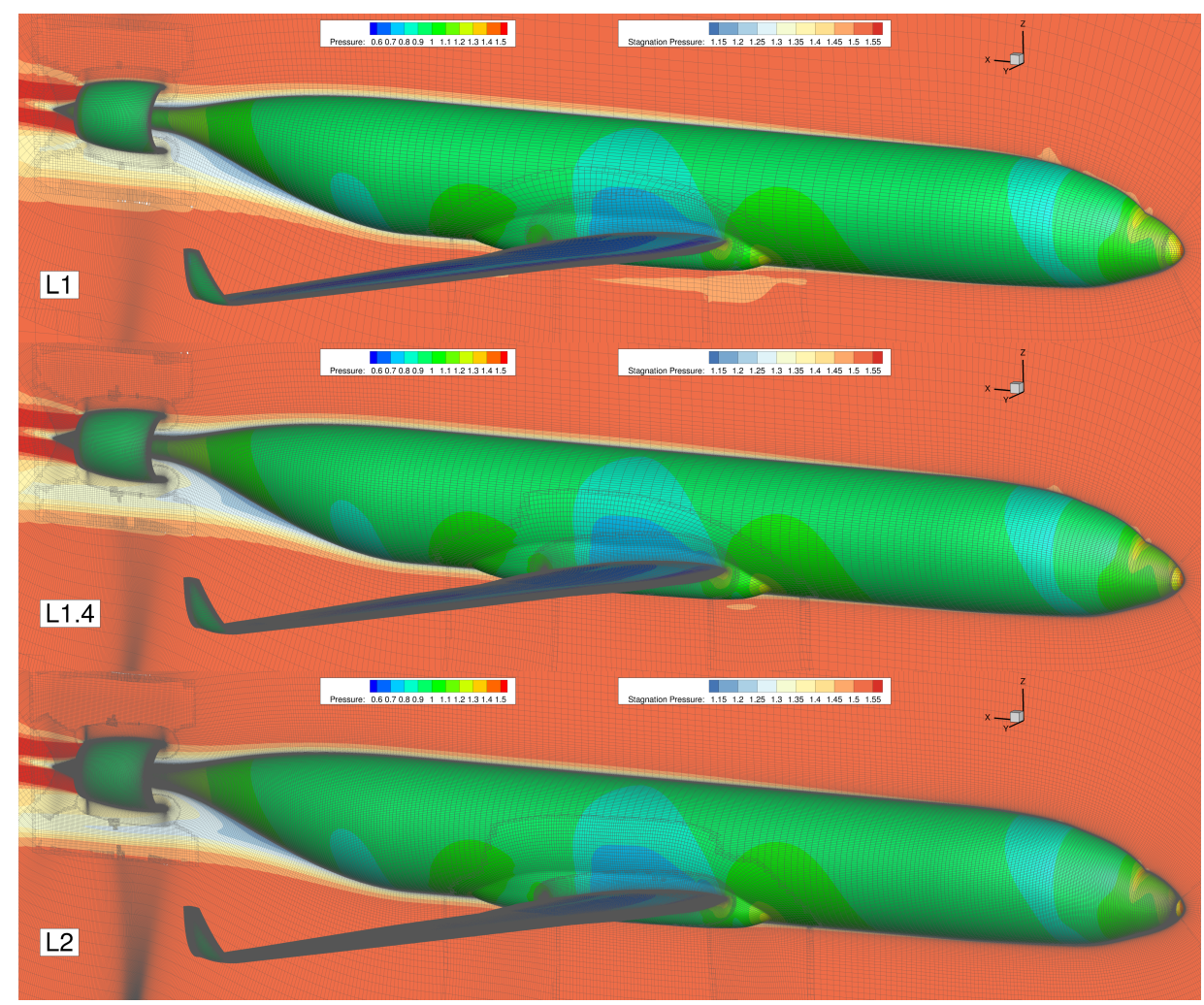

Figure 4: The three mesh levels of the wing-body-duct configuration. Stagnation pressure is shown on the symmetry plane and static pressure on the body.

Four optimizations have been carried out to investigate how fan-face distortion may be minimized on the STARC-ABL configuration. The first two use the body-duct configuration while the last two use the wing-body-duct configuration. For each configuration, both a single point and multi-point optimization are run. The operating conditions for the multipoint optimizations are given in Table 2. The thrust values (an input to the simplified actuator zone model), is set beforehand such that the fan requires approximately 3500 shaft horsepower, which is taken from the systems-level analysis performed by Welstead et al. ${ }^{26}$ Note that the thrust is given for the half-body configuration to match the CFD analysis.

Table 2: Operating conditions

\begin{tabular}{rllll}
\hline $\begin{array}{r}\text { Design } \\
\text { Condition }\end{array}$ & Mach & $\begin{array}{l}\text { Angle of } \\
\text { attack }\left({ }^{\circ}\right)\end{array}$ & $\begin{array}{l}\text { Altitude } \\
(\mathrm{ft})\end{array}$ & $\begin{array}{l}\text { Thrust } \\
(\mathrm{N})\end{array}$ \\
\hline 1 & 0.785 & 0.5 & 36000 & 8500 \\
2 & 0.785 & 2.0 & 36000 & 8500 \\
3 & 0.785 & 3.5 & 36000 & 8500 \\
4 & 0.50 & 5.0 & 10000 & 10800 \\
5 & 0.25 & 8.0 & 0 & 13000 \\
\hline
\end{tabular}

The first three conditions are at the cruise Mach number and altitude. The three angles of attack are used to ensure a robust design across the cruise flight envelope. The two off design conditions, 4 and 5 , represent a climb and takeoff condition respectively. In the absence of another rational approach, each design point is equally weighted for the multipoint optimization. The single point optimizations use just the nominal design condition, $\alpha=2.0^{\circ}$. 


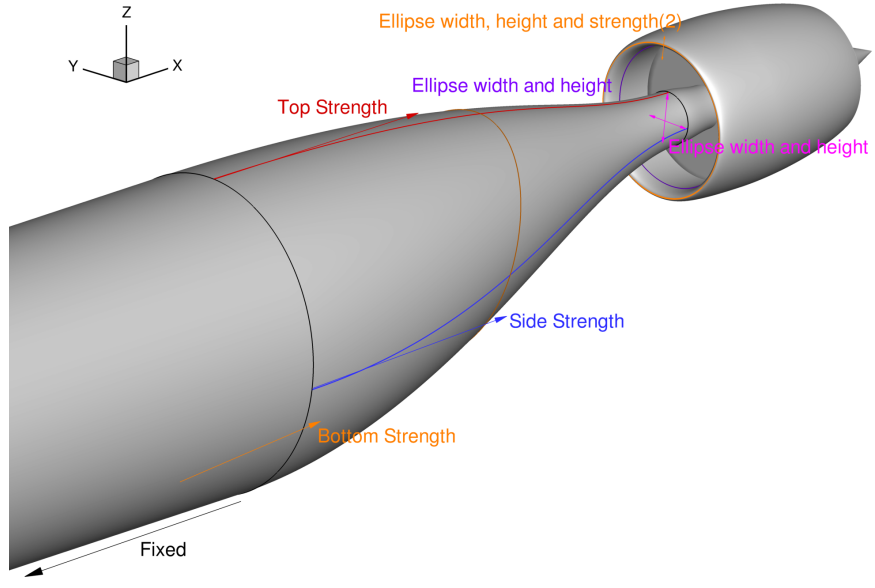

(a) 11 design variables are used to parameterize the STARC-ABL. The nacelle leading edge has two strength design variables

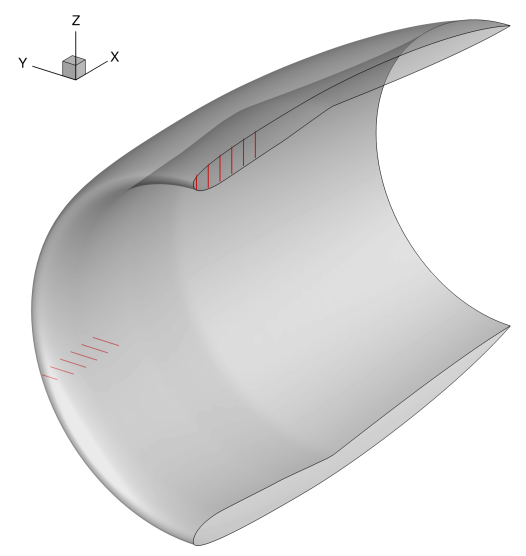

(b) "Toothpick" thickness constraints on the duct leading edge

Figure 5: Design variables and geometric constraints

The optimization problem formulations can be summarized as follows:

$\begin{array}{rll}\text { minimize } & \sum_{k=1}^{N} W_{k} I_{k} & \text { Quantity } \\ \text { with respect to } & \text { Fuselage shape } & 5 \\ & \text { Nacelle shape } & 6 \\ \text { subject to } & C_{D_{i}} \leq C_{D_{i_{0}}}+.0001 & N \\ & t_{j} \geq 0.95 \times t_{j_{\text {init }}} & 10\end{array}$

The $C_{D}$ constraints are formulated such that the design never exceeds more than a 1 count drag penalty relative to the initial design for each flight condition.

\section{Results}

The optimization convergence history for each of the 4 optimization combinations are given in Figure 6 . The optimization algorithm was able to successfully reduce the weighted objective metric for each of the cases. Note that the values plotted in Figure 6 are the uniform weighting of the smoothed objective function, i.e. after applying the KS function over each of the rings. In all other places in the paper where distortion values are given, they are given as the non-smooth maximum over each of the rings. The two cases with the wing (wbd 1pt and wbd 5pt) show the most improvement, but these cases had lower performing starting points.

It can be instructive to examine the total pressure traces for each of the rings at the fan face. This is the raw data that that is used to compute the ARP distortion metric. Figure 7a shows the traces for the body-duct configuration for the baseline and optimized designs while Figure $7 \mathrm{~b}$ shows the same for the wing-body-duct configuration.

The single point body-duct optimization is able to produce exceptionally low distortion levels, especially for rings 2 through 5. This is associated with an increase in the multiple-per-revs number as the large pockets of low total pressure are more even distributed across a larger number of smaller pockets. For the baseline wing-body-duct configuration, there is very large total pressure variation especially on the outer two rings, 4 and 5. This is primarily due to the inlet ingesting nearly free stream air that has not been substantially impacted by either the boundary later or the inviscid diffusion of the fuselage diffuser. It is evident that the $1 \mathrm{pt}$ and $5 \mathrm{pt}$ optimizations produces nearly identical designs with the total pressure traces nearly indistinguishable. As with the body-duct optimization, the multiple-per-rev metric increased with more, but smaller, total pressure pockets as this lowers the ARP1420 metric. For both the 1pt and 5pt optimizations the peak distortion, located on the outermost ring 5 , is reduced from 0.0506 to 0.0222 for the 5 pt design. 


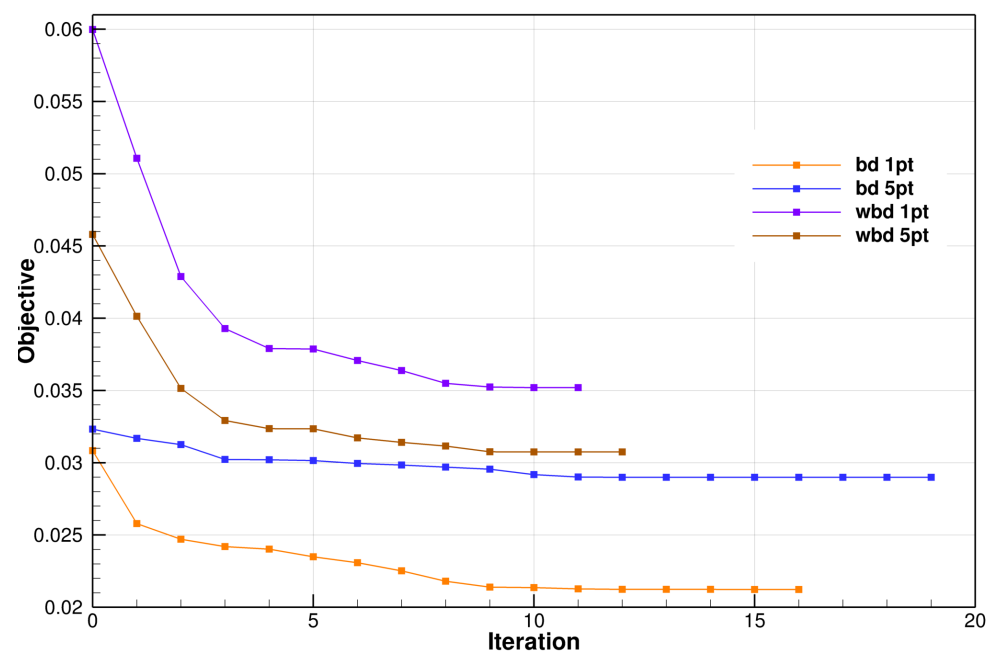

Figure 6: Convergence of the weighted, smoothed KS objective function for each optimization.

A more complete picture of the distortion performance comparison between the designs is given in Figure 8. Here we preform an alpha sweep at the cruise mach and altitude as well as evaluate the two off design (takeoff and climb) conditions for all the configurations. It is immediately clear that the wing downwash has a negative effect on the distortion, especially at the lower angles of attack. The baseline body-duct configuration shows a distortion minimum around $2.5^{\circ}$. The reason for this is due to the offsetting effects of asymmetric boundary layer growth and fuselage diffusion and the asymmetric vertical placement of the duct relative to the fuselage center-line. A similar trend of decreasing distortion with increasing angle of attack is also seen on the baseline wing-body-duct configuration, although no minimum is found between $0^{\circ}$ and $4^{\circ}$. As expected, the body-duct single point optimization has the lowest distortion at the nominal design point of $2^{\circ}$. However, the $5 \mathrm{pt}$ design at $2.75^{\circ}$ shows the lowest measured distortion, but only by a small margin. The 5pt design trades a small performance penalty at low angles of attack to mitigate performance degradation at the larger angles of attack. In both cases, the distortion at the two off-design conditions changed only slightly.

The performance improvement for the wing-body-duct configuration is more significant. Both of the optimized designs lower the distortion over the entire range of cruise angles of attack as well as at the two off-design conditions. Like the body-configuration, the optimized designs have a distortion minimum between $2^{\circ}$ and $3^{\circ}$. Since most of the cruise portion of the flight will take place in this narrow range of angles of attack, it is highly desirable to have the lowest distortion in this region. The distortion at the two off-design conditions improved considerably as well.

A closer look at the changes in the geometry is given in Figure 9. Firstly, the wing-body-duct 1pt and 5 pt configurations are nearly indistinguishable and thus only the 5pt design is shown. This design increase the slope of the fuselage diffuser on the top, while reducing it on the bottom. This helps reduce the high total-pressure pocket near the top of nacelle. A noticeable "bump" is added to the fuselage at the nacelle inlet which potentially helps diffuse the flow into the inlet near the hub, lowering the distortion. The same general changes were made for the body-duct configurations as well, but to a lesser extent. From the top view, we see all configurations reduced the fuselage diffuser angle with the largest change for the wing-bodyduct optimized design. All optimized designs increased the vertical nacelle ellipse axis while reducing the horizontal axis.

Figure 10 shows the contours of total pressure for each configuration at each of the five specified operating conditions. It is easy to confirm that the bd $1 \mathrm{pt}$ optimization at the nominal operating condition of $2^{\circ}$ has a nearly uniform circumferential distribution of total pressure. However, at the $0.5^{\circ}$ degree and $3.5^{\circ}$ conditions, the multipoint $5 \mathrm{pt}$ design performs better. For both the single and multipoint designs, the nacelle inlet is no longer circular but an ellipse which is higher than it is wide.

For the wing-body-duct optimization, the effect of the downwash on the distortion is clear. Comparing the baseline configurations, we see the wing-body-duct configuration has a much larger region of high total pressure air near the top of the nacelle as a direct consequence of the downwash. The optimal nacelle shape 
to mitigate the effects of this distortion is similar to that of the body-duct configuration with a nacelle greater in height than width. However, in the wing-body-duct configuration the fuselage cross section at the nacelle LE has a vertical elliptical cross section which is in contrast to the horizontally elliptical cross sections for the body-duct configuration.

A summary of the key parameters from each of the optimized designs is given in Table 3 . The drag coefficients for the optimized design are within approximately a single drag count as specified by the optimization formulation. This constraint was not active for the 1pt body-duct optimization but was active for design point 3 of the $5 \mathrm{pt}$ optimization. In contrast, for the wing-body-duct optimization, the drag constraints were active for each of the first 3 design points. The power listed in the table refers to the power required to be added to the flow in the actuator zone in order to supply the requested thrust. It is given for the half-body configuration and does not include a fan efficiency factor. Since we did not control the nozzle exit area or the supplied thrust to the actuator region, it was not possible to enforce that the mass flow and flow power remained unchanged. The body-duct configuration saw slight increases in the mass flow overall, while the wing-body-duct configuration saw a slight drop in mass flow for the cruise conditions and a slight increase in mass flow for the off-design conditions. Even though the distortion improvement was made with only a small drag penalty of 1 count or less, the optimizations did not constrain that the power required from under-wing engines to produce the (fixed) thrust did not increase. This increased power requirement translates to higher fuel burn and will be considered in future studies.

All optimized designs increased the required power to varying degrees. The single point body-duct optimization increased the power required by up to $1.87 \%$ while the $5 \mathrm{pt}$ design required only a modest increase of between $.22 \%$ and $0.3 \%$. Power increases for the wing-body-duct design ranged from $0.61 \%$ to $1.39 \%$. While these are not large changes, it does highlight the need for ensuring that the aerodynamic and propulsive models are fully consistent. Any inconsistency between the models can be exploited by the optimizer to improve the objective function while violating a constraint that is not defined in the optimization problem formulation.

Table 3: Data summary for each design

\begin{tabular}{|c|c|c|c|c|c|c|c|c|c|c|c|c|c|}
\hline Config & $\begin{array}{l}\text { Flight } \\
\text { Cond }\end{array}$ & $\begin{array}{l}\text { ARP1420 } \\
\text { Distortion }\end{array}$ & $\begin{array}{l}C_{D} \\
\text { (counts) }\end{array}$ & $\begin{array}{l}\dot{m} \\
(\mathrm{~kg} / \mathrm{s})\end{array}$ & $\begin{array}{l}\text { Power } \\
(\mathrm{MW})\end{array}$ & $\begin{array}{l}\% \text { Change } \\
\text { in Power }\end{array}$ & Config & $\begin{array}{l}\text { Flight } \\
\text { Cond }\end{array}$ & $\begin{array}{l}\text { ARP1420 } \\
\text { Distortion }\end{array}$ & $\begin{array}{l}C_{D} \\
\text { (counts) }\end{array}$ & $\begin{array}{l}\dot{m} \\
(\mathrm{~kg} / \mathrm{s})\end{array}$ & $\begin{array}{l}\text { Power } \\
(\mathrm{MW})\end{array}$ & $\begin{array}{l}\% \text { Change } \\
\text { in Power }\end{array}$ \\
\hline \multirow{5}{*}{$\begin{array}{r}\text { Baseline } \\
\text { bd }\end{array}$} & 1 & 0.0442 & 96.87 & 76.23 & 1.1979 & - & \multirow{5}{*}{$\begin{array}{r}\text { Baseline } \\
\text { wbd }\end{array}$} & 1 & 0.0649 & 194.02 & 76.81 & 1.2004 & - \\
\hline & 2 & 0.0189 & 96.57 & 75.48 & 1.1910 & - & & 2 & 0.0506 & 246.86 & 75.99 & 1.1932 & - \\
\hline & 3 & 0.0148 & 96.86 & 75.42 & 1.1900 & - & & 3 & 0.0390 & 354.32 & 75.34 & 1.1871 & - \\
\hline & 4 & 0.0146 & 80.98 & 170.43 & 1.1314 & - & & 4 & 0.0133 & 272.55 & 168.40 & 1.1184 & - \\
\hline & 5 & 0.0134 & 106.48 & 142.38 & 1.1611 & - & & 5 & 0.0050 & 417.69 & 149.718 & 1.2029 & - \\
\hline \multirow[t]{5}{*}{$\mathrm{bd} 1 \mathrm{pt}$} & 1 & 0.0283 & 96.29 & 76.40 & 1.2114 & 1.13 & \multirow[t]{5}{*}{ wbd $1 p t$} & 1 & 0.0350 & 195.03 & 76.30 & 1.2081 & 0.64 \\
\hline & 2 & 0.0080 & 96.12 & 75.88 & 1.2062 & 1.27 & & 2 & 0.0232 & 247.93 & 75.72 & 1.2022 & 0.75 \\
\hline & 3 & 0.0235 & 96.54 & 76.11 & 1.2081 & 1.52 & & 3 & 0.0220 & 355.43 & 75.34 & 1.1984 & 0.95 \\
\hline & 4 & 0.0204 & 80.88 & 172.61 & 1.1526 & 1.87 & & 4 & 0.0095 & 272.53 & 169.78 & 1.1339 & 1.39 \\
\hline & 5 & 0.0146 & 106.79 & 144.22 & 1.1813 & 1.73 & & 5 & 0.0049 & 416.85 & 150.46 & 1.2116 & 0.73 \\
\hline \multirow[t]{5}{*}{ bd $5 p t$} & 1 & 0.0323 & 97.52 & 76.01 & 1.2006 & 0.22 & \multirow[t]{5}{*}{ wbd 5pt } & 1 & 0.0348 & 195.07 & 76.32 & 1.2077 & 0.61 \\
\hline & 2 & 0.0130 & 97.12 & 75.37 & 1.1937 & 0.22 & & 2 & 0.0229 & 247.99 & 75.73 & 1.2018 & 0.72 \\
\hline & 3 & 0.0151 & 97.94 & 75.18 & 1.1930 & 0.25 & & 3 & 0.0222 & 355.51 & 75.35 & 1.1982 & 0.93 \\
\hline & 4 & 0.0162 & 81.20 & 170.82 & 1.1348 & 0.30 & & 4 & 0.0092 & 272.58 & 169.79 & 1.1339 & 1.39 \\
\hline & 5 & 0.0116 & 107.34 & 142.51 & 1.1640 & 0.25 & & 5 & 0.0045 & 416.23 & 150.58 & 1.2112 & 0.69 \\
\hline
\end{tabular}

\section{Conclusions and Future Work}

A series of four optimizations were presented on simplified STARC-ABL configurations to investigate the ability of using aerodynamic shaping to reduce the fan-face distortion of the aft-boundary layer ingestion fan. The first important observation is that the wing downwash has a significant impact on the fan-face distortion. We saw that the optimized shapes from the body-duct optimization and the wing body duct optimizations differ significantly and thus the wing-downwash is necessary to be included in the optimization. In addition, the best achievable distortion for the wing-body-duct configuration is approximately double the 
body-duct-configuration. It is also possible that slight modifications of wing at the wing root may be able to positively influence the distortion without adversely affecting other aspects of the wing design. Such geometric freedom may be investigated in future work. For the body-duct configuration, we saw a modest effect of the single-point optimization as opposed to the multipoint optimization. This type of behavior is consistent with other types of aerodynamic shape optimizations where a small decrease in performance at a single operating condition can substantially improve performance at other conditions. However, for the wing-body-duct optimizations, there is very little difference between the two designs. This means that shape sensitivities at all the design conditions all point in the same direction, and modifications made at the design point improve all other design points as well. For this case, the main driver of the distortion is the wing downwash and since the optimizer was given little to no control of the downwash, the fuselage and nacelle shapes to mitigate this effect are nearly independent of the design condition.

The unconstrained change in BLI power required highlights the multidisciplinary nature of propulsionairframe integration. Even for the relatively simple optimization problem described in the paper, it is necessary to include the entire nacelle design to ensure consistency with the propulsion model. Future work will involving a much more thorough propulsion model including the under wing engines as well as more geometric detail including the vertical and horizontal stabilizers. This will allow for the evaluation of multidisciplinary trade-offs between the aerodynamic design and propulsion cycle design. ${ }^{8,11}$

\section{Acknowledgments}

This work is partially supported by the Unconventional Propulsion Airframe Integration (UPAI) and Hybrid Gas Electric Propulsion (HGEP) sub-projects of the Advanced Air Transport Technology (AATT) project under NASA's ARMD Advanced Air Vehicles Program (AAVP). Computer time was provided by NASA's Advanced Supercomputing (NAS) division at NASA Ames Research Center. The authors would like thank Jason Welstead, Justin Gray and LAVA group members for valuable discussions on airframe propulsion integration.

\section{References}

${ }^{1}$ Gerald Carrier, Olivier Atinault, Richard Grenon, and Christophe Verbecke. Numerical and experimental aerodynamic investigations of boundary layer ingestion for improving propulsion efficiency of future air transport. In 31st AIAA Applied Aerodynamics Conference, San Diego, California, January 2016.

${ }^{2}$ W. M. Chan. Developments and strategies and software tools for overset structured grid generation and connectivity. In 20th AIAA Computational Fluid Dynamics Conference, Honolulu, Hawaii, June 2011.

${ }^{3}$ W. M. Chan, R. J Gomez, S. E. Rogers, and P. G. Buning. Best practices in overset grid generation. In 32nd AIAA Fluid Dynamics Conference, St. Louis, Missouri, June 2002.

${ }^{4}$ E. M. Greitzer David K Hall and C. S. Tan. Analysis of fan stage conceptual design attributes for boundary layer ingestion. Jounary of Turbomachinery, 139, July 2017.

${ }^{5}$ Mark Drela. Development of the D8 transport configuration. In 29th AIAA Applied Aerodynamics Conference. American Institute of Aeronautics and Astronautics, June 2011.

${ }^{6}$ Alaa A. Elmiligui, William J. Fredericks, Mark D. Guynn, and Richard L. Campbell. Numerical investigation of a fuselage boundary layer ingestion propulsion concept. In 2013 Aviation technology, Integration and Operations Conference, Los Angeles, California, August 2013.

${ }^{7}$ Philip E. Gill, Walter Murray, and Michael A. Saunders. SNOPT: An SQP algorithm for large-scale constrained optimization. SIAM Journal of Optimization, 12(4):979-1006, 2002.

${ }^{8}$ Justin Gray, Jeffrey Chin, Tristan Hearn, Eric Hendricks, Thomas Lavelle, and Joaquim R. R. A. Martins. Chemical equilibrium analysis with adjoint derivatives for propulsion cycle analysis. Journal of Propulsion and Power, 33(5):1041-1052, September 2017.

${ }^{9}$ Alex M. Stoll Gregor Veble Mikic, JoeBen Bevirt, Rok Grah, and Mark D. Moore. Fuselage boundary layer ingestion propulsion applied to a thin haul commuter aircraft for optimal efficiency. In 16th Aviation technology, Integration and Operations Conference, Washington, D.C, June 2016.

${ }^{10}$ Andrew S. Hahn. Vehicle sketch pad: A parametric geometry modeler for conceptual aircraft design. In Proceedings of the 48th AIAA Aerospace Sciences Meeting, Orlando, FL, January 2010.

${ }^{11}$ Dr. Tristan Hearn, Eric Hendricks, Jeffrey Chin, Justin Gray, and Dr. Kenneth T. Moore. Optimization of turbine engine cycle analysis with analytic derivatives. In 17th AIAA/ISSMO Multidisciplinary Analysis and Optimization Conference, part of AIAA Aviation 2016 (Washington, DC), 2016.

${ }^{12} \mathrm{SAE}$ International. Gas turbine inlet flow distortion guidelines. Aerospace Recommended Practice ARP1420, SAE International, 2017.

${ }^{13}$ Gaetan K. W. Kenway, Graeme J. Kennedy, and Joaquim R. R. A. Martins. Scalable parallel approach for high-fidelity steady-state aeroelastic analysis and derivative computations. AIAA Journal, 52(5):935-951, May 2014. 
${ }^{14}$ Gaetan K. W. Kenway and Joaquim R. R. A. Martins. Multipoint high-fidelity aerostructural optimization of a transport aircraft configuration. Journal of Aircraft, 51(1):144-160, January 2014.

${ }^{15}$ Gaetan K. W. Kenway, Ney Secco, Joaquim R. R. A. Martins, Asitav Mishra, and Karthik Duraisamy. An efficient parallel overset method for aerodynamic shape optimization. In Proceedings of the 58th AIAA/ASCE/AHS/ASC Structures, Structural Dynamics, and Materials Conference, AIAA SciTech Forum, Grapevine, TX, January 2017.

${ }^{16}$ H.J.M Kok, Mark Voskuijl, and Michel J. L. van Torren. Distributed propulsion featuring boundary layer ingestion engines for the blended wing body subsonic transport. In 51st AIAA/ASME/ASCE/AHS/ASC Structures, Structural Dynamics, amd Materials Conference, Orlando, Florida, April 2010.

${ }^{17}$ Edward Luke, Eric Collins, and Eric Blades. A fast mesh deformation method using explicit interpolation. Journal of Computational Physics, 231(2):586-601, January 2012.

${ }^{18}$ Zhoujie Lyu, Gaetan K. Kenway, Cody Paige, and Joaquim R. R. A. Martins. Automatic differentiation adjoint of the Reynolds-averaged Navier-Stokes equations with a turbulence model. In 21st AIAA Computational Fluid Dynamics Conference, San Diego, CA, Jul. 2013.

${ }^{19}$ Charles A. Mader, Joaquim R. R. A. Martins, Juan J. Alonso, and Edwin van der Weide. ADjoint: An approach for the rapid development of discrete adjoint solvers. AIAA Journal, 46(4):863-873, April 2008.

${ }^{20}$ Robert A McDonald. Advanced modeling in openvsp. In 16h AIAA Aviation Technology, Integration, and Operations Conference, AIAA AVIATION Forum, June 2016.

${ }^{21}$ Ruben E. Perez, Peter W. Jansen, and Joaquim R. R. A. Martins. pyOpt: A Python-based object-oriented framework for nonlinear constrained optimization. Structural and Multidisciplinary Optimization, 45(1):101-118, January 2012.

${ }^{22}$ A. P. Plas, M. A. Sargeant, V. Madani, D. Crichton, and E. M. Greitzer. Performance of a boundary layer ingesting (bli) propulsion system. In 45th AIAA Aerospace Sciences Meeting and Exhibit, Reno, Nevada, Januaray 2007.

${ }^{23} \mathrm{P}$. Spalart and S. Allmaras. A one-equation turbulence model for aerodynamic flows. In 30th Aerospace Sciences Meeting and Exhibit, 1992

${ }^{24}$ Alejandra Urango, Mark Drea, Edward M. Greitzer, Neil A. Titchener, Michael K. Lieu, Nina M. Siu, Arthur C. Huang, gregory M. Gatlin, and Judith A. Hannon. Preliminary experimental assessment of the boundary layer ingestion belefit for the d8 aircraft. In 52nd AIAA Aerospace Sciences Meeting, National Harbor, Maryland, January 2014.

${ }^{25}$ E. van der Weide, G. Kalitzin, J. Schluter, and J. J. Alonso. Unsteady turbomachinery computations using massively parallel platforms. In Proceedings of the 44th AIAA Aerospace Sciences Meeting and Exhibit, Reno, NV, 2006. AIAA $2006-0421$.

${ }^{26}$ Jason R. Welstead and James L. Felder. Conceptual design of a single-aisle turboelectric commercial transport with fuselage boundary layer ingestion. In 54th AIAA Aerospace Sciences Meeting, San Diego, California, January 2016.

${ }^{27}$ Ludovic Wiart, Olivier Atinault, Bernard Paluch, David Hue, and Richard Grenon. Development of nova aircraft configurations for large engine integration studies. In 33rd AIAA Applied Aerodynamics Conference, Dallas, Texas, June 2015.

${ }^{28}$ Gregory A. Wrenn. An indirect method for numerical optimization using the Kreisselmeier-Steinhauser function. Technical Report CR-4220, NASA Langley Research Center, Hampton, VA, 1989. 

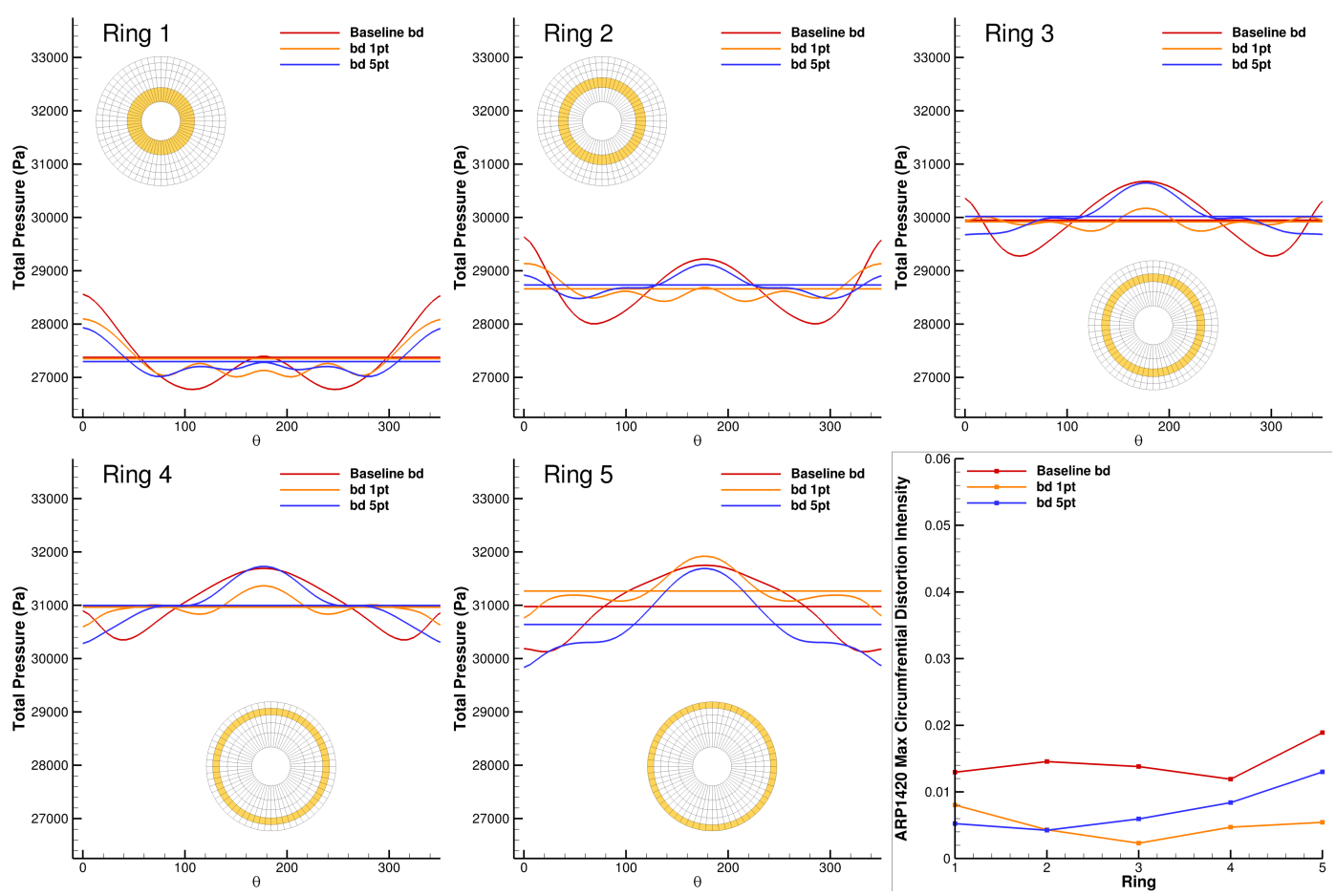

(a) Total pressure traces for the body-duct configuration
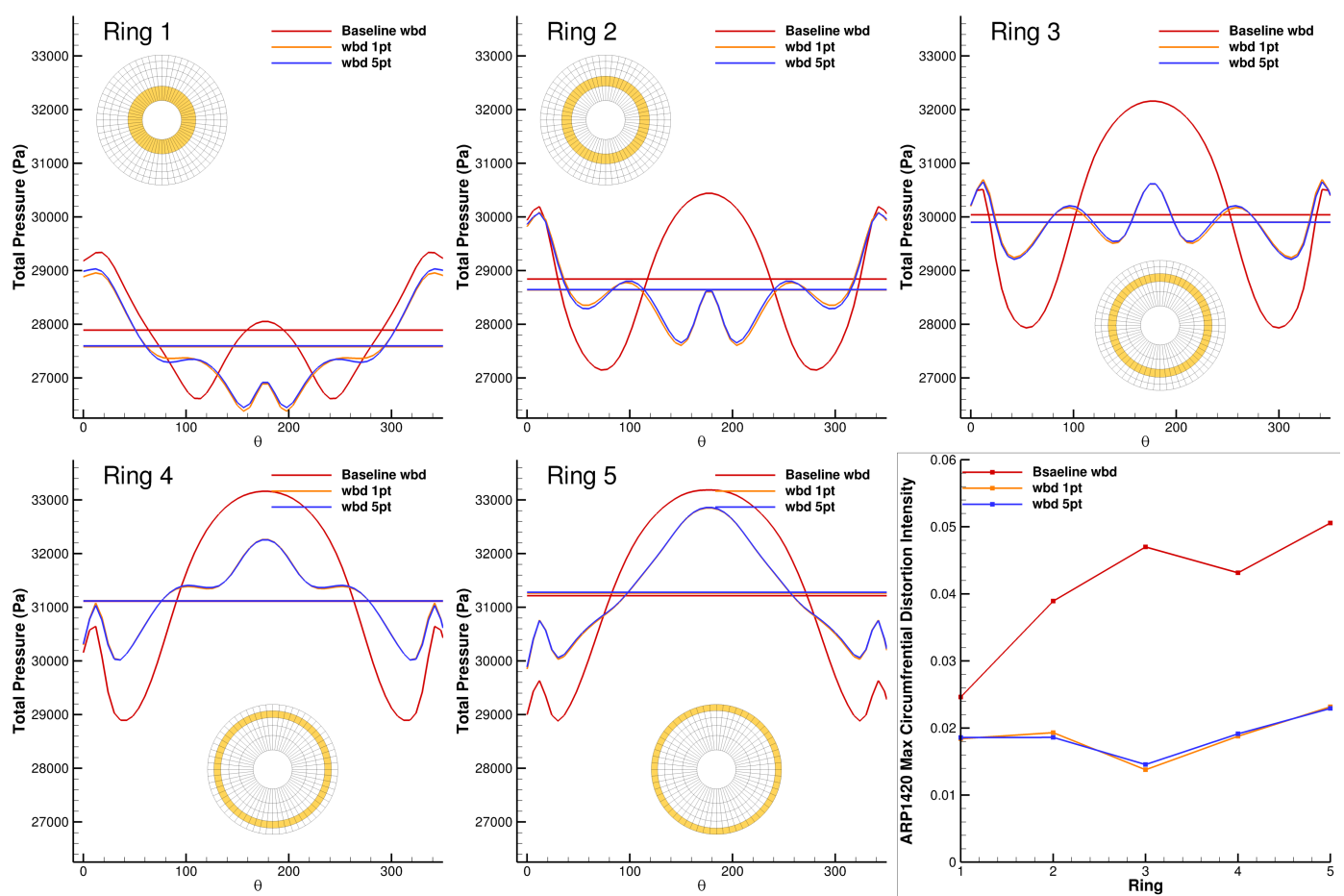

(b) Total pressure traces for the wing-body-duct configuration

Figure 7: Total pressure traces for baseline and optimized configurations at the nominal operating condition (Condition 2) 

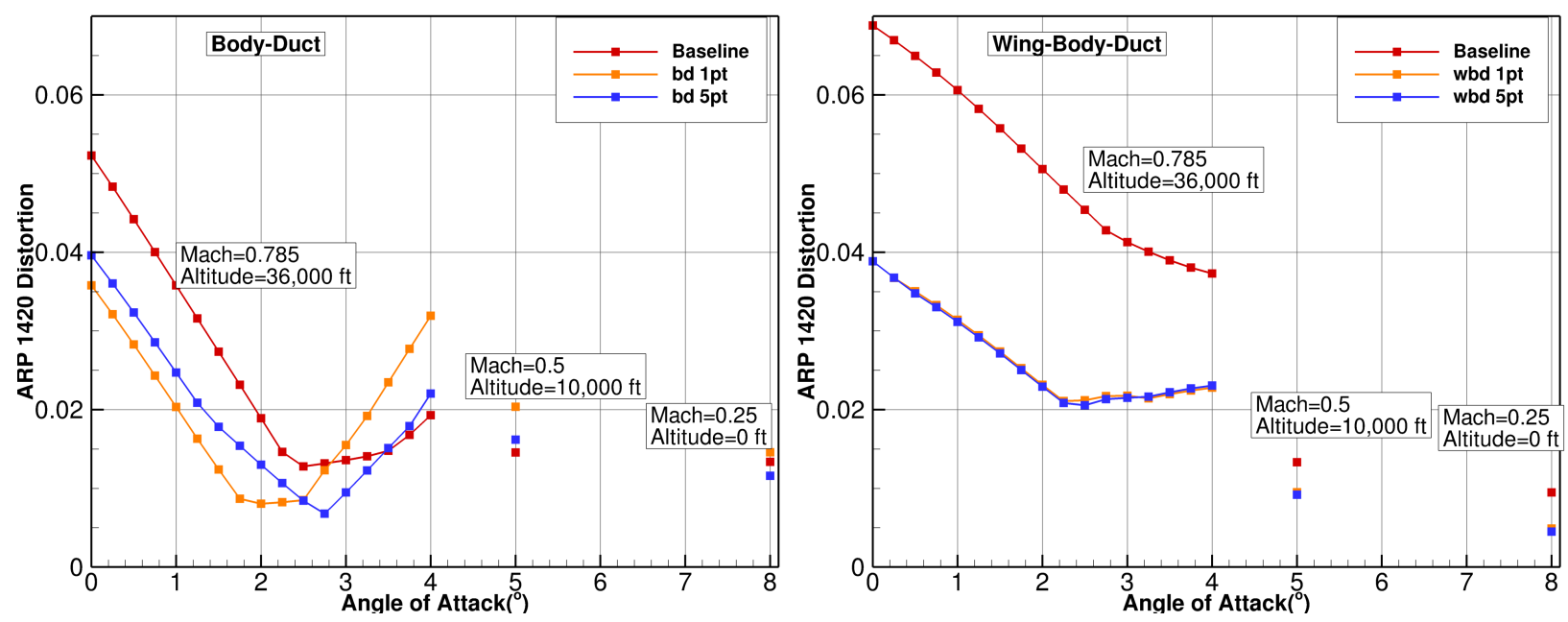

Figure 8: Maximum ARP1420 distortion for the baseline and optimized designs.
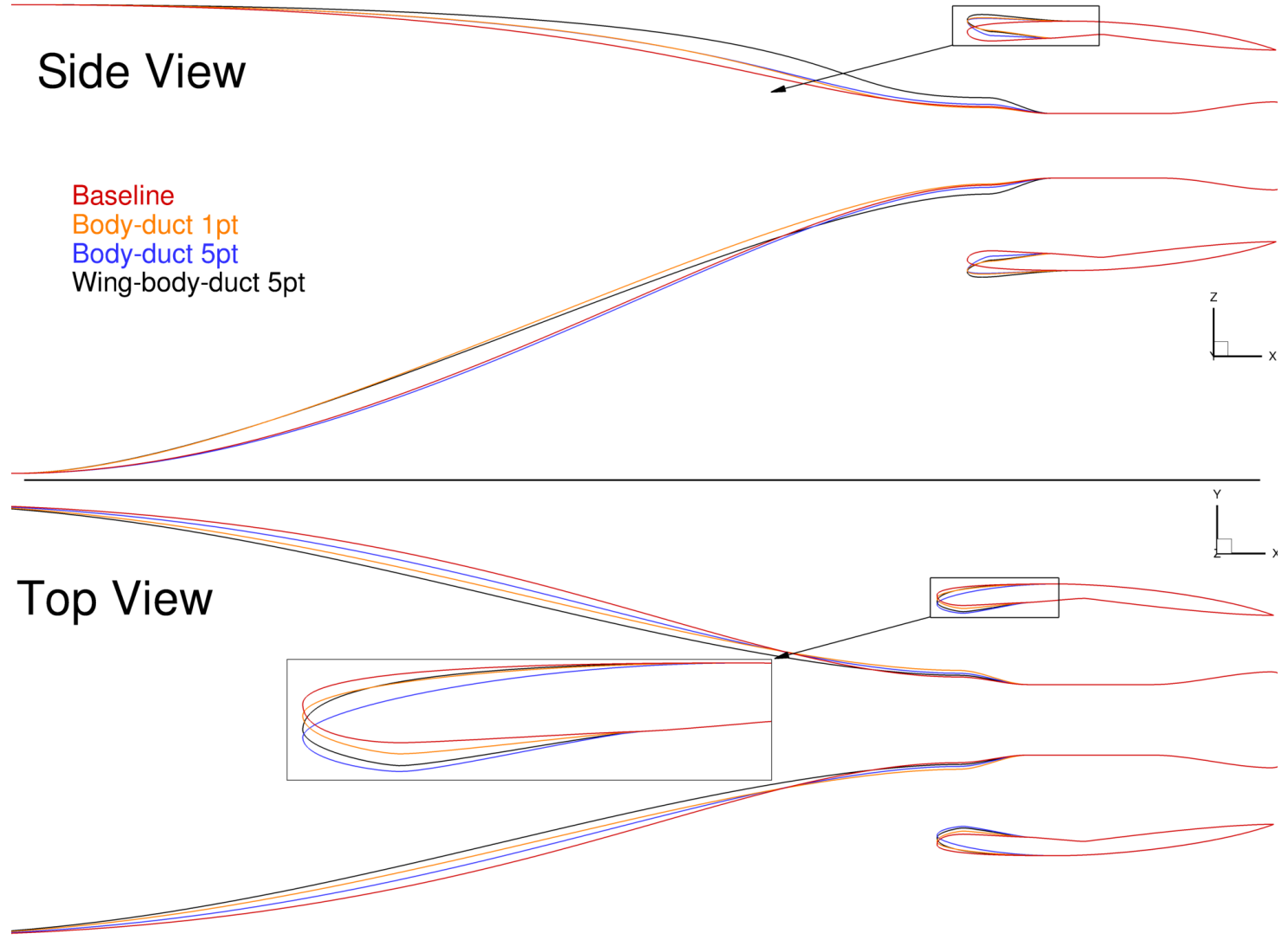

Figure 9: Comparison of the body contour lines at the symmetry plane (top) and the horizontal mid-plane of the nacelle (bottom). 


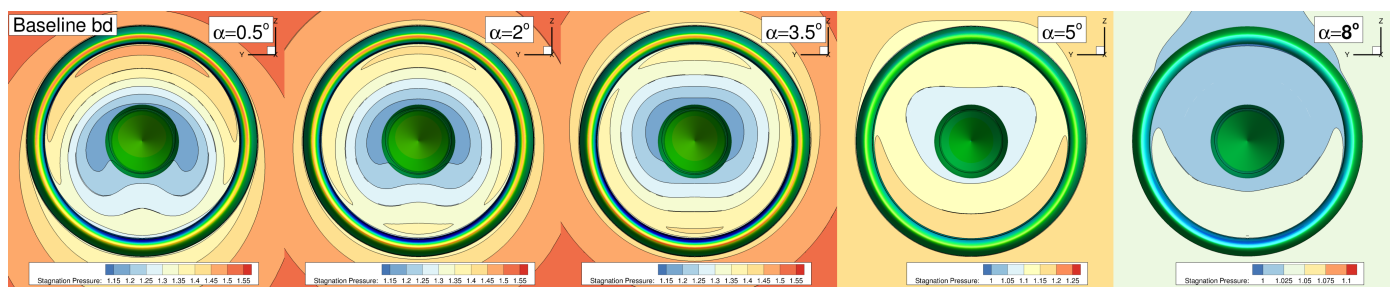

(a) Baseline Body-Duct

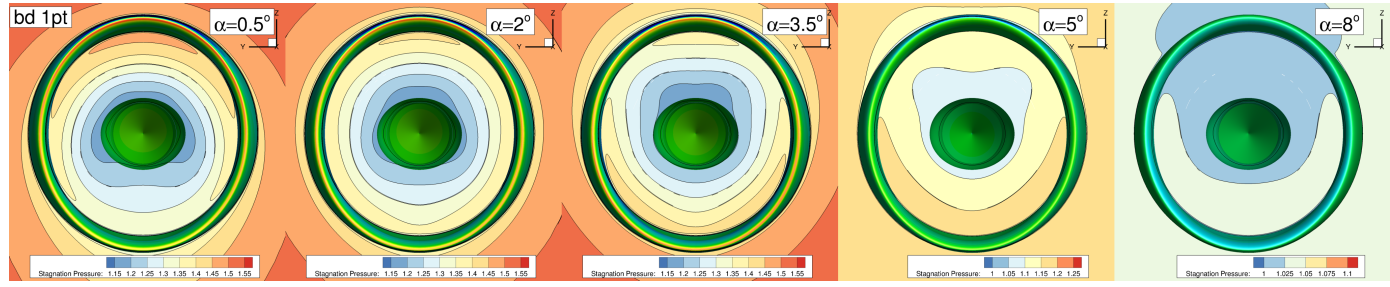

(b) Body-Duct 1pt Optimization

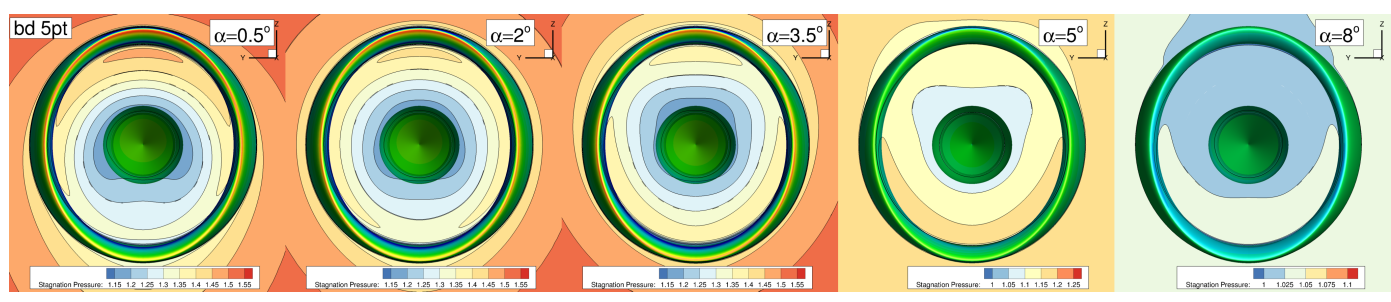

(c) Body-Duct 5pt Optimization

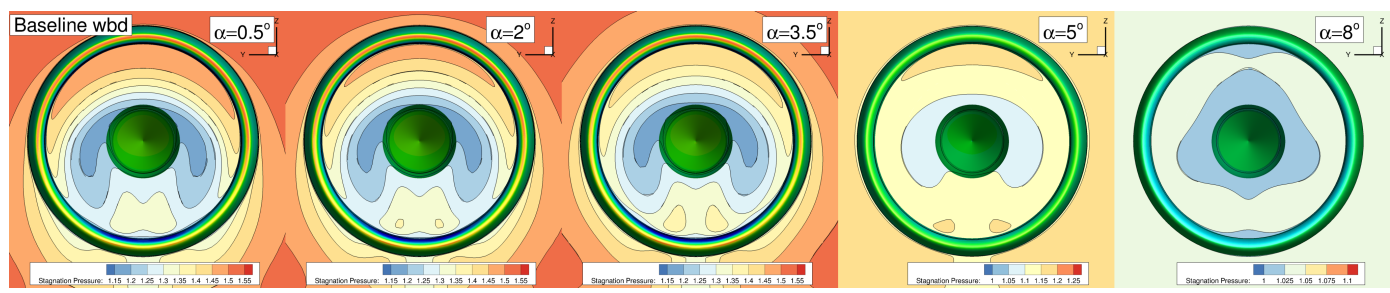

(d) Baseline Wing-Body-Duct

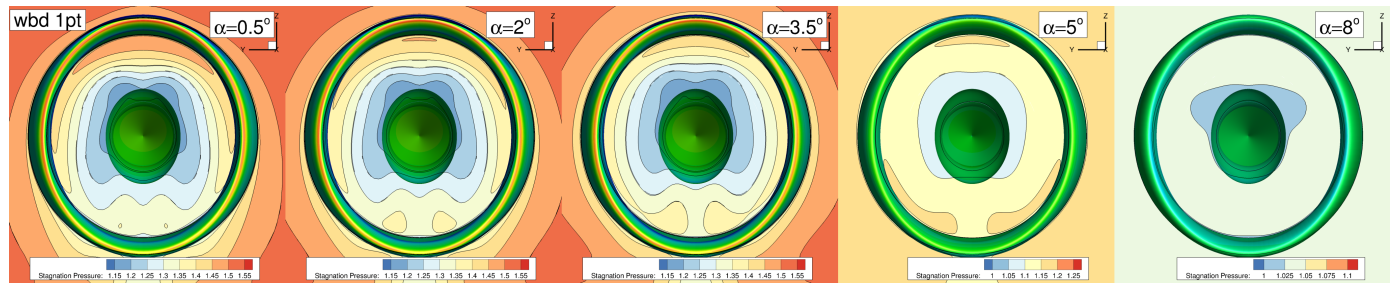

(e) Wing-Body-Duct 1pt Optimization

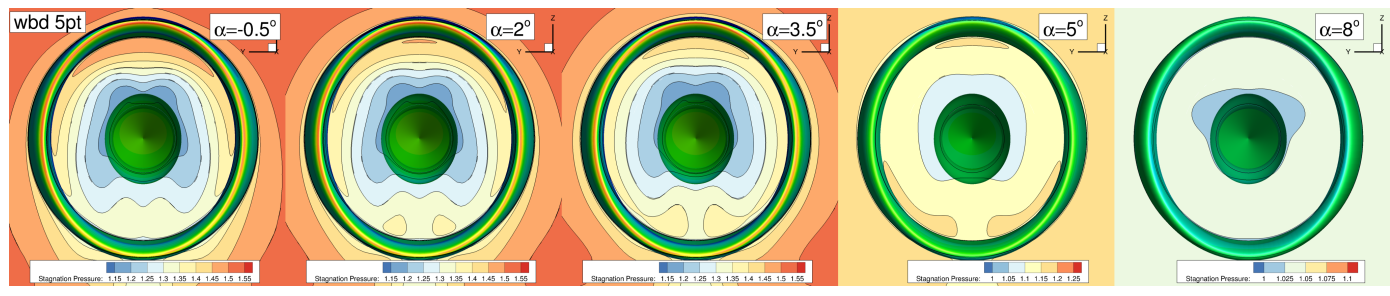

(f) Wing-Body-Duct 5pt Optimization

Figure 10: Total pressure contours for each design at each operating condition 